\title{
Oscillation Theorems for Second-Order Forced Neutral Nonlinear Differential Equations with Delayed Argument
}

\author{
Jing Shao ${ }^{1,2}$ and Fanwei Meng ${ }^{2}$ \\ ${ }^{1}$ Department of Mathematics, Jining University, Qufu, Shandong 273155, China \\ 2 School of Mathematical Sciences, Qufu Normal University, Qufu, Shandong 273165, China \\ Correspondence should be addressed to Jing Shao, shaojing99500@163.com \\ Received 13 October 2009; Accepted 14 December 2009 \\ Academic Editor: Elena Braverman
}

Copyright (c) 2010 J. Shao and F. Meng. This is an open access article distributed under the Creative Commons Attribution License, which permits unrestricted use, distribution, and reproduction in any medium, provided the original work is properly cited.

We are concerned with the oscillation of the forced second-order neutral nonlinear differential equations with delayed argument in the form $\left(r(t)(x(t)+a(t) x(\sigma(t)))^{\prime}\right)^{\prime}+p(t) f(x(\tau(t)))+$ $\sum_{i=1}^{n} q_{i}(t)|x(t)|^{\lambda_{i}} \operatorname{sng} x(t)=e(t)$. No restriction is imposed on the potentials $p(t), q_{i}(t)$, ande $(t)$ to be nonnegative. Our methodology is somewhat different from those of previous authors.

\section{Introduction}

In this paper, we study the oscillatory behavior of the forced neutral nonlinear functional differential equation of the form

$$
\left(r(t)(x(t)+a(t) x(\sigma(t)))^{\prime}\right)^{\prime}+p(t) f(x(\tau(t)))+\sum_{i=1}^{n} q_{i}(t)|x(t)|^{\lambda_{i}} \operatorname{sgn} x(t)=e(t)
$$

where $t \geq t_{0}$. In this paper, we assume that

$\left(I_{1}\right) r(t) \in C\left(\left[t_{0}, \infty\right),(0, \infty)\right), r^{\prime}(t) \geq 0, \int^{\infty}(1 / r(t)) d t=\infty$,

$\left(I_{2}\right) a(t) \in C\left(\left[t_{0}, \infty\right),[0,1)\right)$,

$\left(I_{3}\right) \sigma(t) \in C\left(\left[t_{0}, \infty\right), \mathbb{R}\right)$ is nondecreasing, $\sigma(t) \leq t$ for $t \geq t_{0}$, and $\lim _{t \rightarrow \infty} \sigma(t)=\infty$,

$\left(I_{4}\right) \tau(t) \in C\left(\left[t_{0}, \infty\right), \mathbb{R}\right), \tau(t) \leq t$ for $t \geq t_{0}$ and $\lim _{t \rightarrow \infty} \tau(t)=\infty$,

$\left(I_{5}\right) p(t), q_{i}(t)$, and $e(t)$ are continuous functions defined on $[0, \infty), p(t)>0, \lambda_{1}>\cdots>$ $\lambda_{m}>1>\lambda_{m+1}>\cdots>\lambda_{n}>0(n>m \geq 1)$,

( $\left.I_{6}\right) f(x)$ is nondecreasing, $f(x) / x \geq M>0$, and $x \neq 0$. 
No restriction is imposed on the potentials $p(t), q_{i}(t)$, and $e(t)$ to be nonnegative. As usual, a solution of (1.1) is called oscillatory if it is defined on some ray $[T, \infty)$ with $T \geq 0$ and has unbounded set of zeros. (1.1) is called oscillatory if all of its solutions on some ray are oscillatory.

In the last decades, there has been an increasing interest in obtaining sufficient conditions for the oscillation and/or nonoscillation of second-order linear and nonlinear delay differential equations (see, for example, [1-22] and the references therein). Let us consider the familiar forced Emden-Fowler equation

$$
x^{\prime \prime}(t)+p(t)|x(t)|^{\lambda} \operatorname{sgn} x(t)=e(t), \quad t \geq t_{0} .
$$

When $\lambda_{1}>1$, (1.2) is known as the superlinear equation, and when $0<\lambda_{1}<1$, it is known as the sublinear equation. The oscillation of (1.2) has been the subject of much attention during the last 50 years; see the seminal book by Agarwal, et al. [23]. Here, we refer to the papers [13 ] and the references cited therein. In this case, one can usually establish oscillation criteria for more general nonlinear equations by using a technique introduced by Kartsatos [9] where it is additionally assumed that $f$ is the second derivative of an oscillatory function. This approach has been expressed in $[5,6]$. Sun $[4]$ has extended these results to delay differential equations of the form of (1.2), where $\lambda \geq 1$ and the potentials $p(t)$ and $e(t)$ are allowed to change sign. However, Sun [4] does not say anything else for the oscillation of equation (1.2) with $0<\lambda<1$. Later, employing the arguments in [4], Çakmak and Tiryaki [7] have established similar oscillation criteria for the equation of the form

$$
x^{\prime \prime}(t)+q(t) f(x(\tau(t)))=e(t)
$$

where $f(x)$ is assumed to satisfy certain growth conditions.

Very recently, Sun et al. $[13,14]$ obtained some new oscillation criteria for the equations in the form

$$
\begin{gathered}
\left(r(t) x^{\prime}(t)\right)^{\prime}+p(t) x(t)+\sum_{i=1}^{n} q_{i}(t)|x(t)|^{\lambda_{i}} \operatorname{sgn} x(t)=0, \\
\left(r(t) x^{\prime}(t)\right)^{\prime}+p(t) x(t)+\sum_{i=1}^{n} q_{i}(t)|x(t)|^{\lambda_{i}} \operatorname{sgn} x(t)=e(t),
\end{gathered}
$$

where $\lambda_{1}>\cdots>\lambda_{m}>1>\lambda_{m+1}>\cdots>\lambda_{n}>0(n>m \geq 1)$. He also established oscillation theorems when $n>1$. When $n=1$, this approach was initiated by Agarwal and Grace [1, pages 244-249] for higher-order equations and subsequently developed in papers of $\mathrm{Ou}$ and Wong [15], Q.Yang [18], X.Yang [19], as well as Sun and Agarwal [16, 17].

In [24], $\mathrm{Xu}$ and Meng studied the oscillation of the equation

$$
\left(r(t)(x(t)+a(t) x(\sigma(t)))^{\prime}\right)^{\prime}+\sum_{i=1}^{n} q_{i}(t) f_{i}\left(y\left(\tau_{i}(t)\right)\right)=0, \quad t \geq t_{0}
$$

by using the generalized Riccati technique and the function class $y$. 
The purpose of this paper is to give some new oscillation criteria for (1.1), which can be regarded as further investigation for the (1.1) including the papers of Sun and Wong [13], $\mathrm{Xu}$ and Meng [24]. These criteria do not assume that $r(t), p(t), q_{i}(t)$, and $e(t)$ are of definite sign. Our methodology is somewhat different from those of previous authors, and the results we obtained are more general than those of Sun and Wong [13].

\section{Main Results}

We will need the following lemmas that have been proved in [13].

Lemma 2.1 (see [13]). Let $\lambda_{i}, i=1,2, \ldots, n$, be $n$-tuple satisfying $\lambda_{1}>\cdots>\lambda_{m}>1>\lambda_{m+1}>$ $\cdots>\lambda_{n}>0$. Then there exists an $n$-tuple $\left(k_{1}, k_{2}, \ldots, k_{n}\right)$ satisfying

$$
\sum_{i=1}^{n} \lambda_{i} k_{i}=1
$$

which also satisfies either

$$
\sum_{i=1}^{n} k_{i}<1, \quad 0<k_{i}<1
$$

or

$$
\sum_{i=1}^{n} k_{i}=1, \quad 0<k_{i}<1
$$

Lemma 2.2 (see [13]). Let $u, A, B, C$, and $D$ be positive real numbers. Then

$$
\begin{aligned}
& \text { (i) } A u^{\alpha}+B \geq \alpha(\alpha-1)^{1 / \alpha-1} A^{1 / \alpha} B^{1-1 / \alpha} u, \alpha>1 \text {, } \\
& \text { (ii) } C u-D u^{\alpha} \geq(\alpha-1) \alpha^{\alpha /(1-\alpha)} C^{\alpha /(\alpha-1)} D^{1 /(1-\alpha)}, 0<\alpha<1 \text {. }
\end{aligned}
$$

Remark 2.3. For a given set of exponents $\lambda_{i}$ satisfying $\lambda_{1}>\cdots>\lambda_{m}>1>\lambda_{m+1}>\cdots>\lambda_{n}>0$, Lemma 2.1 ensures the existence of an $n$-tuple $\left(k_{1}, k_{2}, \ldots, k_{n}\right)$ such that either $(a)$ and $(b)$ hold or $(a)$ and $(c)$ hold. When $n=2$ and $\lambda_{1}>1>\lambda_{2}>0$, in the first case, we have that

$$
k_{1}=\frac{1-\lambda_{2}\left(1-k_{0}\right)}{\lambda_{1}-\lambda_{2}}, \quad k_{2}=\frac{\lambda_{1}\left(1-k_{0}\right)-1}{\lambda_{1}-\lambda_{2}}
$$

where $k_{0}$ can be any positive number satisfying $0<k_{0}<\left(\lambda_{1}-1\right) / \lambda_{1}$. This will ensure that $0<k_{1}, k_{2}<1$, and conditions $(a)$ and $(b)$ are satisfied. In the second case, we simply solve (a) and (c) and obtain

$$
k_{1}=\frac{1-\lambda_{2}}{\lambda_{1}-\lambda_{2}}, \quad k_{2}=\frac{\lambda_{1}-1}{\lambda_{1}-\lambda_{2}}
$$


Theorem 2.4. Suppose that, for any $T \geq 0$, there exist constants $a_{1}, b_{1}, a_{2}, b_{2}$ such that $T \leq a_{1}<$ $b_{1} \leq a_{2}<b_{2}$, and

$$
\begin{aligned}
q_{i}(t) \geq 0, \quad t & \in\left[\tau\left(a_{1}\right), b_{1}\right] \cup\left[\tau\left(a_{2}\right), b_{2}\right], i=1, \ldots, n, \\
& e(t) \leq 0, \quad t \in\left[\tau\left(a_{1}\right), b_{1}\right] \\
& e(t) \geq 0, \quad t \in\left[\tau\left(a_{2}\right), b_{2}\right] .
\end{aligned}
$$

Let $D\left(a_{j}, b_{j}\right)=\left\{u \in C^{1}\left[a_{j}, b_{j}\right]: u^{v+1}>0, v>0\right.$ is a constant, $t \in\left(a_{j}, b_{j}\right)$, and $u\left(a_{j}\right)=$ $\left.u\left(b_{j}\right)=0\right\}$, for $j=1,2$. Assume that there exists a positive, nondecreasing function $\rho \in C^{1}\left(\left[t_{0}, \infty\right), \mathbb{R}\right)$ such that, for some $H \in D\left(a_{j}, b_{j}\right)$ and for some $\theta \geq 1$,

$$
\int_{a_{j}}^{b_{j}}\left[H^{v+1}(t) \rho(t) R(t)-\frac{\theta \rho(t) r(t) H^{\nu-1}(t) A^{2}(t)}{4}\right] d t>0
$$

for $j=1,2$, then (1.1) is oscillatory, where

$$
\begin{gathered}
R(t)=M p(t)[1-a(\tau(t))] \frac{\tau(t)-\tau\left(a_{j}\right)}{t-\tau\left(a_{j}\right)}+a_{0}(1-a(t))|e(t)|^{k_{0}} \prod_{i=1}^{n} q_{i}^{k_{i}}(t), \\
A(t)=H(t) \frac{\rho^{\prime}(t)}{\rho(t)}+(v+1) H^{\prime}(t)
\end{gathered}
$$

$a_{0}=\prod_{i=0}^{n} k_{i}^{-k_{i}}$, and $k_{0}, k_{1}, \ldots, k_{n}$ are positive constants satisfying $(a)$ and $(b)$ of Lemma 2.1.

Proof. Assume to the contrary that there exists a solution $x(t)$ of (1.1) such that $x(t)>$ $0, x(\tau(t))>0, x(\sigma(t))>0$, when $t \geq t_{0}>0$, for some $t_{0}$ depending on the solution $x(t)$. Set

$$
z(t)=x(t)+a(t) x(\sigma(t))
$$

By assumption, we have that $z(t)>0$ for $t \geq t_{0} \geq 0$, and from (2.4) it follows that

$$
\left(r(t) z^{\prime}(t)\right)^{\prime}=e(t)-p(t) f(x(\tau(t)))-\sum_{i=1}^{n} q_{i}(t)|x(t)|^{\lambda_{i}} \operatorname{sgn} x(t) \leq 0, \quad t \geq t_{0} \geq 0 .
$$

It is not difficult to show that $z^{\prime}(t)$ is eventually positive. In fact, first, we know that $z^{\prime}(t) \not \equiv 0$ for sufficiently large $t$, since $z(t)$ is nontrivial. Second, if there exists an $t_{1} \geq t_{0}$ such that $r\left(t_{1}\right) z^{\prime}\left(t_{1}\right)=C<0$, then $r(t) z^{\prime}(t) \leq C$ for $t \geq t_{1} \geq t_{0}$, that is, $z^{\prime}(t) \leq C / r(t)$, and hence, $z(t) \leq z\left(t_{1}\right)+\int_{t_{1}}^{t}(C / r(t)) d t \rightarrow-\infty$ as $t \rightarrow \infty$, which contradicts the fact that $z(t)>0$. Without loss of generality; say $z^{\prime}(t)>0, t \geq t_{0} \geq 0$. Thus we have that

$$
x(t) \geq z(t)-a(t) z(t), \quad t \geq t_{0} \geq 0 .
$$


Define

$$
w(t)=\rho(t) \frac{r(t) z^{\prime}(t)}{z(t)}, \quad t \geq t_{0}
$$

It follows from (2.7) that $w(t)$ satisfies the following differential equality:

$$
w^{\prime}(t)=\frac{\rho^{\prime}(t)}{\rho(t)} w(t)-\rho(t) \frac{p(t) f(x(\tau(t)))}{z(t)}-\frac{w^{2}(t)}{\rho(t) r(t)}+\rho(t) \frac{e(t)}{z(t)}-\frac{\rho(t) \sum_{i=1}^{n} q_{i}(t) x^{\lambda_{i}}(t)}{z(t)}
$$

Using (2.6), we have that

$$
\begin{aligned}
w^{\prime}(t) \leq & \frac{\rho^{\prime}(t)}{\rho(t)} w(t)-\rho(t) \frac{p(t) f((1-a(\tau(t))) z(\tau(t)))}{z(t)}-\frac{w^{2}(t)}{\rho(t) r(t)} \\
& +\rho(t) \frac{e(t)}{z(t)}-\rho(t) \sum_{i=1}^{n} q_{i}(t)(1-a(t))^{\lambda_{i}} z^{\lambda_{i}-1}(t)
\end{aligned}
$$

and by the condition $f(x) / x \geq M>0$, we have that

$$
\begin{aligned}
w^{\prime}(t) \leq & \frac{\rho^{\prime}(t)}{\rho(t)} w(t)-M \rho(t) p(t) \frac{(1-a(\tau(t))) z(\tau(t))}{z(t)}-\frac{w^{2}(t)}{\rho(t) r(t)} \\
& +\rho(t) \frac{e(t)}{z(t)}-\rho(t) \sum_{i=1}^{n} q_{i}(t)(1-a(t))^{\lambda_{i}} z^{\lambda_{i}-1}(t) .
\end{aligned}
$$

By assumption, we can choose $a_{1}, b_{1} \geq t_{0}$ such that $b_{1} \geq \tau\left(a_{1}\right), \tau^{2}\left(a_{1}\right)=\tau\left(\tau\left(a_{1}\right)\right) \geq t_{0}, q_{i}(t) \geq$ $0, i=1,2, \ldots, n$, for $t \in\left[\tau\left(a_{1}\right), b_{1}\right]$, and $e(t) \leq 0$ for $t \in\left[\tau\left(a_{1}\right), b_{1}\right]$. Recall the arithmeticgeometric mean inequality (see [25])

$$
\sum_{i=0}^{n} k_{i} u_{i} \geq \prod_{i=0}^{n} u_{i}^{k_{i}}, \quad u_{i} \geq 0
$$

where $k_{0}=1-\sum_{i=1}^{n} k_{i}$ and $k_{i}>0, i=1,2, \ldots, n$ are chosen to satisfy $(a)$ and $(b)$ of Lemma 2.1 for the given $\lambda_{1}, \lambda_{2}, \ldots, \lambda_{n}>0$. Now return to (2.10) and identify $u_{0}=k_{0}^{-1}|e(t)| z^{-1}(t)$ and $u_{i}=k_{i}^{-1} q_{i}(t) z^{\lambda_{i}-1}(t)(1-a(t))^{\lambda_{i}}$ in $(2.11)$ to obtain

$$
\begin{aligned}
w^{\prime}(t) \leq & \frac{\rho^{\prime}(t)}{\rho(t)} w(t)-M \rho(t) p(t) \frac{(1-a(\tau(t))) z(\tau(t))}{z(t)} \\
& -\frac{w^{2}(t)}{\rho(t) r(t)}-\rho(t)(1-a(t)) k_{0}^{-k_{0}}|e(t)|^{k_{0}} \prod_{i=1}^{n} k_{i}^{-k_{i}} q_{i}^{k_{i}}(t) .
\end{aligned}
$$


From (1.1), we can easily obtain $z^{\prime \prime}(t) \leq 0$, for $t \in\left[\tau\left(a_{1}\right), b_{1}\right]$. Therefore, we have that, for $t \in\left[\tau\left(a_{1}\right), b_{1}\right]$,

$$
z(t)-z\left(\tau\left(a_{1}\right)\right)=z^{\prime}(s)\left(t-\tau\left(a_{1}\right)\right) \geq z^{\prime}(t)\left(t-\tau\left(a_{1}\right)\right) .
$$

Noting that $z(t)>0$ for $t \geq \tau\left(a_{1}\right)$, we get by (2.13) that

$$
z(t) \geq z^{\prime}(t)\left(t-\tau\left(a_{1}\right)\right), \quad t \in\left[\tau\left(a_{1}\right), b_{1}\right]
$$

that is,

$$
\frac{z^{\prime}(t)}{z(t)} \leq \frac{1}{t-\tau\left(a_{1}\right)}, \quad t \in\left[\tau\left(a_{1}\right), b_{1}\right]
$$

Integrating (2.15) from $\tau(t)$ to $t>a_{1}$, we obtain

$$
\frac{z(\tau(t))}{z(t)} \geq \frac{\tau(t)-\tau\left(a_{1}\right)}{t-\tau\left(a_{1}\right)}, \quad t \in\left(a_{1}, b_{1}\right]
$$

By using (2.16) in (2.12), we have that, for $t \in\left(a_{1}, b_{1}\right]$,

$$
\begin{aligned}
w^{\prime}(t) \leq & \frac{\rho^{\prime}(t)}{\rho(t)} w(t)-M \rho(t) p(t)[1-a(\tau(t))] \frac{\tau(t)-\tau\left(a_{1}\right)}{t-\tau\left(a_{1}\right)} \\
& -\frac{w^{2}(t)}{\rho(t) r(t)}-\rho(t)(1-a(t)) k_{0}^{-k_{0}}|e(t)|^{k_{0}} \prod_{i=1}^{n} k_{i}^{-k_{i}} q_{i}^{k_{i}}(t) \\
= & \frac{\rho^{\prime}(t)}{\rho(t)} w(t)-\rho(t) R(t)-\frac{w^{2}(t)}{\rho(t) r(t)}
\end{aligned}
$$

Multiplying both sides of (2.17) by $H^{v+1}(t)$ as given in the hypothesis of Theorem 2.4 and integrating (2.17) from $a_{1}$ to $b_{1}$, we obtain

$$
\int_{a_{1}}^{b_{1}} H^{v+1}(t) \rho(t) R(t) d t \leq \int_{a_{1}}^{b_{1}} H^{v+1}(t) \frac{\rho^{\prime}(t)}{\rho(t)} w(t) d t-\int_{a_{1}}^{b_{1}} H^{v+1}(t) w^{\prime}(t) d t-\int_{a_{1}}^{b_{1}} H^{v+1}(t) \frac{w^{2}(t)}{\rho(t) r(t)} d t .
$$

Using the integration by parts formula, we have that

$$
\begin{aligned}
\int_{a_{1}}^{b_{1}} H^{v+1}(t) w^{\prime}(t) d t & =\left.H^{v+1}(t) w(t)\right|_{a_{1}} ^{b_{1}}-\int_{a_{1}}^{b_{1}}(v+1) H^{v}(t) H^{\prime}(t) w(t) d t \\
& =-\int_{a_{1}}^{b_{1}}(v+1) H^{v}(t) H^{\prime}(t) w(t) d t
\end{aligned}
$$


where $H\left(a_{1}\right)=H\left(b_{1}\right)=0$. Substituting (2.19) into (2.18), we obtain

$$
\begin{aligned}
\int_{a_{1}}^{b_{1}} H^{v+1}(t) \rho(t) R(t) d t \leq & \int_{a_{1}}^{b_{1}} H^{v+1}(t) \frac{\rho^{\prime}(t)}{\rho(t)} w(t) d t+\int_{a_{1}}^{b_{1}}(v+1) H^{v}(t) H^{\prime}(t) w(t) d t \\
& -\int_{a_{1}}^{b_{1}} H^{v+1}(t) \frac{w^{2}(t)}{\rho(t) r(t)} d t \\
& =\int_{a_{1}}^{b_{1}} A(t) H^{v}(t) w(t) d t-\int_{a_{1}}^{b_{1}} H^{v+1}(t) \frac{w^{2}(t)}{\rho(t) r(t)} d t
\end{aligned}
$$

Then

$$
\begin{aligned}
\int_{a_{1}}^{b_{1}} H^{v+1}(t) \rho(t) R(t) d t \leq & -\int_{a_{1}}^{b_{1}}\left[-A(t) H^{v}(t) w(t)+H^{v+1}(t) \frac{w^{2}(t)}{\rho(t) r(t)}\right] d t \\
= & -\int_{a_{1}}^{b_{1}}\left[\sqrt{\frac{H^{v+1}(t)}{\theta \rho(t) r(t)}} w(t)-\sqrt{\frac{\theta \rho(t) r(t)}{4 H^{v+1}(t)}} H^{v}(t) A(t)\right]^{2} d t \\
& +\int_{a_{1}}^{b_{1}}\left[\sqrt{\frac{\theta \rho(t) r(t)}{4 H^{v+1}(t)}} H^{v}(t) A(t)\right]^{2} d t-\int_{a_{1}}^{b_{1}} \frac{(\theta-1) H^{v+1}(t)}{\theta \rho(t) r(t)} w^{2}(t) d t .
\end{aligned}
$$

From the hypothesis of Theorem 2.4 and (2.21), we have that

$$
\begin{aligned}
\int_{a_{i}}^{b_{i}} & {\left[H^{v+1}(t) \rho(t) R(t)-\left(\sqrt{\frac{\theta \rho(t) r(t)}{4 H^{v+1}(t)}} H^{v}(t) A(t)\right)^{2}\right] d t } \\
= & \int_{a_{i}}^{b_{i}}\left[H^{v+1}(t) \rho(t) R(t)-\frac{\theta \rho(t) r(t) H^{v-1}(t) A^{2}(t)}{4}\right] d t \leq 0,
\end{aligned}
$$

which contradicts (2.2). When $x(t)$ is eventually negative, we can obtain similar contradiction using the interval $\left[\tau\left(a_{2}\right), b_{2}\right]$ instead of $\left[\tau\left(a_{1}\right), b_{1}\right]$. This completes the proof.

Remark 2.5. Let $r(t)=1, q_{i}(t)=0$, and $a(t)=0$ for $i=1,2, \ldots, n$. It is easy to see that Theorem 2.4 reduces to Theorem 1 of [7].

In Theorem 2.6, we do not impose any restriction on signs of those coefficients corresponding to sublinear terms of $(1.1)$, that is, $q_{l}(t)$ for $l=m+1, \ldots, n$. If it is nonpositive, we can easily see that Theorem 2.4 is invalid. However, the following theorem is valid for this case. 
Theorem 2.6. Suppose that, for any $T \geq 0$, there exist constants $a_{1}, b_{1}, a_{2}, b_{2}$ such that $T \leq a_{1}<$ $b_{1} \leq a_{2}<b_{2}$ and

$$
\begin{aligned}
q_{i}(t) \geq 0, \quad t & \in\left[\tau\left(a_{1}\right), b_{1}\right] \cup\left[\tau\left(a_{2}\right), b_{2}\right], i=1, \ldots, m, \\
& e(t)<0, \quad t \in\left[\tau\left(a_{1}\right), b_{1}\right] \\
& e(t)>0, \quad t \in\left[\tau\left(a_{2}\right), b_{2}\right] .
\end{aligned}
$$

Assume that there exists a positive, nondecreasing function $\rho \in C^{1}\left(\left[t_{0}, \infty\right), \mathbb{R}\right)$ such that, for some $H \in D\left(a_{j}, b_{j}\right)$ and for some $\theta \geq 1$,

$$
\int_{a_{j}}^{b_{j}}\left[H^{v+1}(t) \rho(t) \bar{R}(t)-\frac{\theta \rho(t) r(t) H^{v-1}(t) A^{2}(t)}{4}\right] d t>0,
$$

for $j=1,2$, then (1.1) is oscillatory, where

$$
\begin{gathered}
A(t)=H(t) \frac{\rho^{\prime}(t)}{\rho(t)}+(v+1) H^{\prime}(t) \\
\bar{R}(t)=p(t) M[1-a(\tau(t))] \frac{\tau(t)-\tau\left(a_{j}\right)}{t-\tau\left(a_{j}\right)}+\sum_{i=1}^{m} \mu_{i}\left(\beta_{i}|e(t)|\right)^{1-1 / \lambda_{i}} q_{i}^{1 / \lambda_{i}}(t) \\
-\sum_{l=m+1}^{n} \gamma_{l}\left(\delta_{l}|e(t)|\right)^{1-1 / \lambda_{l}} \bar{q}_{l}^{1 / \lambda_{l}}(t)
\end{gathered}
$$

with $\sum_{i=1}^{m} \beta_{i}+\sum_{l=m+1}^{n} \delta_{l}=1$ for $\beta_{i}>0, \delta_{l}>0, \mu_{i}=\lambda_{i}\left(\lambda_{i}-1\right)^{1 / \lambda_{i}-1}, i=1, \ldots, m, \gamma_{l}=\lambda_{l}(1-$ $\left.\lambda_{l}\right)^{1 / \lambda_{l}-1}$, and $\bar{q}_{l}(t)=\max \left\{-q_{l}(t), 0\right\}, l=m+1, \ldots, n$.

Proof. Assume to the contrary that there exists a solution $x(t)$ of (1.1) such that $x(t)>$ $0, x(\tau(t))>0$, when $t \geq t_{0}>0$, for some $t_{0}$ depending on the solution $x(t)$. When $x(t)$ is eventually negative, the proof follows the same argument using the interval $\left[\tau\left(a_{2}\right), b_{2}\right]$ instead of $\left[\tau\left(a_{1}\right), b_{1}\right]$. Apply the assumption of $\beta_{i}$ and $\delta_{l}$, then (1.1) is rearranged as

$$
\begin{aligned}
& \left(r(t)(x(t)+a(t) x(\sigma(t)))^{\prime}\right)^{\prime}+p(t) f(x(\tau(t))) \\
& \quad+\sum_{i=1}^{m}\left[q_{i}(t)|x(t)|^{\lambda_{i}}-\beta_{i} e(t)\right]+\sum_{l=m+1}^{n}\left[q_{l}(t)|x(t)|^{\lambda_{l}}-\delta_{l} e(t)\right]=0 .
\end{aligned}
$$

Noting the assumption (2.23) and applying Lemma 2.2(i) to the first summation term in (2.26), we get that

$$
\begin{aligned}
& \left(r(t)(x(t)+a(t) x(\sigma(t)))^{\prime}\right)^{\prime}+p(t) f(x(\tau(t)))+x(t) \sum_{i=1}^{m} \mu_{i}\left(\beta_{i}|e(t)|\right)^{1-1 / \lambda_{i}} q_{i}^{1 / \lambda_{i}}(t) \\
& \quad+\sum_{l=m+1}^{n}\left(q_{l}(t)|x(t)|^{\lambda_{l}}-\delta_{l} e(t)\right) \leq 0 .
\end{aligned}
$$


Introduce the Riccati substitution as (2.4) and apply Lemma 2.2(ii) to each of the nonlinear terms in the last sum in (2.27). Here $u=x(t), \alpha=\lambda_{l}, D=\bar{q}_{l}(t)$, and

$$
C=\alpha(1-\alpha)^{1 / \alpha-1}\left(\delta_{l}|e(t)|\right)^{1-1 / \alpha} \bar{q}_{l}^{1 / \alpha}(t) .
$$

We can obtain from (2.27) the following Riccati inequality:

$$
\begin{aligned}
w^{\prime}(t) \leq & \frac{\rho^{\prime}(t)}{\rho(t)} w(t)-\rho(t) p(t) M[1-a(\tau(t))] \frac{\tau(t)-\tau\left(a_{1}\right)}{t-\tau\left(a_{1}\right)}-\frac{w^{2}(t)}{\rho(t) r(t)} \\
& -\rho(t) \sum_{i=1}^{m} \mu_{i}\left(\beta_{i}|e(t)|\right)^{1-1 / \lambda_{i}} q_{i}^{1 / \lambda_{i}}(t)+\rho(t) \sum_{l=m+1}^{n} \gamma_{l}\left(\delta_{l}|e(t)|\right)^{1-1 / \lambda_{l}} \bar{q}_{l}^{1 / \lambda_{l}}(t) \\
= & \frac{\rho^{\prime}(t)}{\rho(t)} w(t)-\rho(t) \bar{R}(t)-\frac{w^{2}(t)}{\rho(t) r(t)}
\end{aligned}
$$

where $\bar{q}_{l}(t)=\max \left\{-q_{l}(t), 0\right\}$. The remaining argument is the same as that in Theorem 2.4 and this completes the proof.

Remark 2.7. Let $f(x)=x, a(t)=0$, and $\tau(t)=t, n=1$. Theorems 2.4 and 2.6 reduce to Theorem 1 of $[8,11]$.

We will use the function class $y$ to study the oscillatory of (1.1). We say that a function $\Phi=\Phi(t, s, l)$ belongs to the function class $y$, denoted by $\Phi \in \mathcal{y}$ if $\Phi \in C(E, \mathbb{R})$, where $E=$ $\left\{(t, s, l): t_{0} \leq l \leq s \leq t<\infty\right\}$, which satisfies $\Phi(t, t, l)=0$, and $\Phi(t, l, l)=0, \Phi(t, s, l) \neq 0$ for $l<s<t$, and has the partial derivative $\partial \Phi / \partial s$ on $E$ such that $\partial \Phi / \partial s$ is locally integrable with respect to $s$ in $E$.

We defined the operator $B[\cdot ; l, t]$ by

$$
B[g ; l, t]=\int_{l}^{t} \Phi^{2}(t, s, l) g(s) d s, \quad \text { for } t \geq s \geq l \geq t_{0}, g(s) \in C\left[t_{0}, \infty\right),
$$

and the function $\varphi=\varphi(t, s, l)$ is defined by

$$
\frac{\partial \Phi(t, s, l)}{\partial s}=\varphi(t, s, l) \Phi(t, s, l)
$$

It is easy to verify that $B[; ; l, t]$ is a linear operator and satisfies

$$
B\left[g^{\prime} ; l, t\right]=-2 B[g \varphi ; l, t], \quad \text { for } g \in C^{1}\left[t_{0}, \infty\right) .
$$

Theorem 2.8. Suppose that, for any $T \geq 0$, there exist constants $a_{1}, b_{1}, a_{2}, b_{2}$ such that $T \leq a_{1}<$ $b_{1} \leq a_{2}<b_{2}$ and satisfies (2.1). Assume that there exists a function $\Phi \in \mathcal{Y}$, such that, for each $t \geq t_{0}$ and for some $\theta \geq 1$,

$$
\limsup _{t \rightarrow \infty} B\left[\rho(s) R(s)-\frac{\theta \rho(s) r(s)}{4}\left(2 \varphi-\frac{\rho^{\prime}(s)}{\rho(s)}\right)^{2} ; t_{0}, t\right]>0,
$$


for $j=1,2$, then (1.1) is oscillatory, where the operator B is defined by $(2.30), \varphi=\varphi\left(t, s, t_{0}\right)$ is defined by $(2.31)$,

$$
R(s)=M p(s)[1-a(\tau(s))] \frac{\tau(s)-\tau\left(a_{j}\right)}{s-\tau\left(a_{j}\right)}+a_{0}(1-a(s))|e(s)|^{k_{0}} \prod_{i=1}^{n} q_{i}^{k_{i}}(s),
$$

$a_{0}=\prod_{i=0}^{n} k_{i}^{-k_{i}}$, and $k_{0}, k_{1}, \ldots, k_{n}$ are positive constants satisfying $(a)$ and $(b)$ of Lemma 2.1.

Proof. We proceed as in Theorem 2.4. Assume to the contrary that there exists a solution $x(t)$ of (1.1) such that $x(t)>0, x(\tau(t))>0$, when $t \geqslant t_{0}>0$, for some $t_{0}$ depending on the solution $x(t)$. From the proof of Theorem 2.4, we obtain (2.16) for all $t \in\left[\tau\left(a_{1}\right), b_{1}\right]$. Applying $B\left[; t_{0}, t\right],\left(t \geq t_{0}\right)$ to $(2.17)$, we have that

$$
B\left[w^{\prime}(s) ; t_{0}, t\right] \leq-B\left[\frac{\rho^{\prime}(s)}{\rho(s)} w(s) ; t_{0}, t\right]-B\left[\rho(s) R(s) ; t_{0}, t\right]-B\left[\frac{w^{2}(s)}{\rho(s) r(s)} ; t_{0}, t\right] .
$$

By (2.32) and above inequality, we have, for $t \geq t_{0}$, that

$$
\begin{aligned}
B\left[\rho(s) R(s) ; t_{0}, t\right] \leq & 2 B\left[w(s) \varphi ; t_{0}, t\right]-B\left[\frac{\rho^{\prime}(s)}{\rho(s)} w(s) ; t_{0}, t\right]-B\left[\frac{w^{2}(s)}{\rho(s) r(s)} ; t_{0}, t\right] \\
=B & -\left(\frac{w(s)}{\sqrt{\theta \rho(s) r(s)}}-\sqrt{\theta \rho(s) r(s)}\left(\varphi-\frac{\rho^{\prime}(s)}{2 \rho(s)}\right)\right)^{2} \\
& \left.+\frac{\theta \rho(s) r(s)}{4}\left(2 \varphi-\frac{\rho^{\prime}(s)}{\rho(s)}\right)^{2}-\frac{\theta-1}{\theta} \frac{w^{2}(s)}{\rho(s) r(s)} ; t_{0}, t\right] \\
\leq B & {\left[\frac{\theta \rho(s) r(s)}{4}\left(2 \varphi-\frac{\rho^{\prime}(s)}{\rho(s)}\right)^{2} ; t_{0}, t\right] . }
\end{aligned}
$$

That is,

$$
B\left[\rho(s) R(s)-\frac{\theta \rho(s) r(s)}{4}\left(2 \varphi-\frac{\rho^{\prime}(s)}{\rho(s)}\right)^{2} ; t_{0}, t\right] \leq 0
$$

Taking the super limit in the above inequality, we have that

$$
\limsup _{t \rightarrow \infty} B\left[\rho(s) R(s)-\frac{\theta \rho(s) r(s)}{4}\left(2 \varphi-\frac{\rho^{\prime}(s)}{\rho(s)}\right)^{2} ; t_{0}, t\right] \leq 0,
$$

which contradicts assumption (2.33). When $x(t)$ is eventually negative, the proof follows the same argument using the interval $\left[\tau\left(a_{2}\right), b_{2}\right]$ instead of $\left[\tau\left(a_{1}\right), b_{1}\right]$. This completes the proof. 
Theorem 2.9. Suppose that, for any $T \geq 0$, there exist constants $a_{1}, b_{1}, a_{2}, b_{2}$ such that $T \leq a_{1}<$ $b_{1} \leq a_{2}<b_{2}$ and satisfies (2.23). Assume that there exists a function $\Phi \in \boldsymbol{y}$ such that, for each $t \geq t_{0}$ and for some $\theta \geq 1$,

$$
\limsup _{t \rightarrow \infty} B\left[\rho(s) \bar{R}(s)-\frac{\theta \rho(s) r(s)}{4}\left(2 \varphi-\frac{\rho^{\prime}(s)}{\rho(s)}\right)^{2} ; t_{0}, t\right]>0
$$

for $j=1,2$, then (1.1) is oscillatory, where the operator B is defined by $(2.30), \varphi=\varphi\left(t, s, t_{0}\right)$ is defined by (2.31), and $\bar{R}(t)$ is defined by (2.25).

The proof of Theorem 2.9 can be completed by following the proofs of Theorems 2.6 and 2.8 with suitable changes, we omit it here.

\section{Corollaries}

As Theorems 2.4-2.9 are rather general, it is convenient for applications to derive a number of oscillation criteria with the appropriate choice of the functions $H, \rho$, and $\Phi(t, s, l)$.

With an appropriate choice of the functions $H(t), \rho(t)$, one can derive from Theorems 2.4 and 2.6 a number of oscillation criteria for (1.1). For example, we consider the simple case $n=2$; hence,$\lambda_{1}>1>\lambda_{2}>0$. Let $b_{j}=a_{j}+\pi / \sqrt{c_{j}}, b_{j}=a_{j}+\pi / 4 \sqrt{d_{j}}, j=1,2$, where $a_{j}, b_{j}$ are given in Theorem 2.4. This determines $c_{j}, d_{j}, j=1,2$. Choose $H(t)=\sin \left[\sqrt{c_{j}}\left(t-a_{j}\right)\right]$, $\rho(t)=\sin ^{k}\left[\sqrt{d_{j}}\left(t-a_{j}\right)\right]$, and $k$ being natural number. We obtain from Theorems 2.4 and 2.6 the following corollaries.

Corollary 3.1. Let $k_{0}, k_{1}, k_{2}$ be chosen to satisfy $(a)$, (b) of Lemma 2.1 for $\lambda_{1}>1>\lambda_{2}>0$. If for any $T \geq t_{0}$, there exist constants $a_{1}, b_{1}, a_{2}, b_{2}$ such that $T \leq a_{1}<b_{1} \leq a_{2}<b_{2}$, then it holds that

$$
\begin{aligned}
& q_{1}(t) \geq 0, \quad t \in\left[\tau\left(a_{1}\right), b_{1}\right] \cup\left[\tau\left(a_{2}\right), b_{2}\right], \\
& q_{2}(t) \geq 0, \\
& e(t) \leq 0, \quad t \in\left[\tau\left(a_{1}\right), b_{1}\right], \\
& e(t) \geq 0, \quad t \in\left[\tau\left(a_{2}\right), b_{2}\right],
\end{aligned}
$$

and for $c_{j}=\pi^{2} /\left(b_{j}-a_{j}\right)^{2}, 16 d_{j}=\pi^{2} /\left(b_{j}-a_{j}\right)^{2}$, and $\theta \geq 1$, one has that

$$
\begin{gathered}
\int_{a_{j}}^{b_{j}}\left[\left(p(t) M(1-a(\tau(t))) \frac{\tau(t)-\tau\left(a_{j}\right)}{t-\tau\left(a_{j}\right)}+k_{0}^{-k_{0}} k_{1}^{-k_{1}} k_{2}^{-k_{2}}|e(t)|^{k_{0}} q_{1}^{k_{1}}(t) q_{2}^{k_{2}}(t)(1-a(t))\right)\right. \\
\times \sin ^{v+1}\left[\sqrt{c_{j}}\left(t-a_{j}\right)\right] \sin ^{k}\left[\sqrt{d_{j}}\left(t-a_{j}\right)\right]-\frac{\theta r(t) c_{j} \sin ^{v-1}\left[\sqrt{c_{j}}\left(t-a_{j}\right)\right]}{4 \sin ^{k}\left[\sqrt{d_{j}}\left(t-a_{j}\right)\right]}
\end{gathered}
$$




$$
\begin{aligned}
& \times\left(k \sqrt{d_{j}} \sin \left[\sqrt{c_{j}}\left(t-a_{j}\right)\right] \sin ^{k-1}\left[\sqrt{d_{j}}\left(t-a_{j}\right)\right] \cos \left[\sqrt{d_{j}}\left(t-a_{j}\right)\right]\right. \\
& \left.\left.+(v+1) \sqrt{c_{j}} \sin ^{k}\left[\sqrt{d_{j}}\left(t-a_{j}\right)\right] \cos \left[\sqrt{c_{j}}\left(t-a_{j}\right)\right]\right)^{2}\right] d t>0,
\end{aligned}
$$

then all solutions of the equation

$$
\begin{aligned}
& \left(r(t)(x(t)+a(t) x(\sigma(t)))^{\prime}\right)^{\prime}+p(t) f(x(\tau(t))) \\
& \quad+q_{1}(t)|x(t)|^{\lambda_{1}} \operatorname{sgn} x(t)+q_{2}(t)|x(t)|^{\lambda_{2}} \operatorname{sgn} x(t)=e(t)
\end{aligned}
$$

are oscillatory.

Corollary 3.2. Assume that, for any $T \geq 0$, there exist constants $a_{1}, b_{1}, a_{2}, b_{2}$ such that $T \leq a_{1}<$ $b_{1} \leq a_{2}<b_{2}$ and

$$
\begin{gathered}
q_{1}(t) \geq 0, \quad t \in\left[\tau\left(a_{1}\right), b_{1}\right] \cup\left[\tau\left(a_{2}\right), b_{2}\right], \\
e(t)<0, \quad t \in\left[\tau\left(a_{1}\right), b_{1}\right], \\
e(t)>0, \quad t \in\left[\tau\left(a_{2}\right), b_{2}\right] .
\end{gathered}
$$

Also assume that, for $\lambda_{1}>1>\lambda_{2}>0$, and that there exists some $\lambda \in(0,1)$ such that

$$
\begin{aligned}
\int_{a_{j}}^{b_{j}}\left[\left(p(t) M(1-a(\tau(t))) \frac{\tau(t)-\tau\left(a_{j}\right)}{t-\tau\left(a_{j}\right)}+\lambda_{1}\left(\lambda_{1}-1\right)^{1 / \lambda_{1}-1}(\lambda|e(t)|)^{1-1 / \lambda_{1}} q_{1}^{1 / \lambda_{1}}(t)(1-a(t))\right.\right. \\
\left.\quad-\lambda_{2}\left(1-\lambda_{2}\right)^{1 / \lambda_{2}-1}((1-\lambda)|e(t)|)^{1-1 / \lambda_{2}} \bar{q}_{2}^{1 / \lambda_{2}}(t)(1-a(t))\right) \\
\quad \times \sin ^{v+1}\left[\sqrt{c_{j}}\left(t-a_{j}\right)\right] \sin ^{k}\left[\sqrt{d_{j}}\left(t-a_{j}\right)\right]-\frac{\theta r(t) c_{j} \sin ^{v-1}\left[\sqrt{c_{j}}\left(t-a_{j}\right)\right]}{4 \sin ^{k}\left[\sqrt{d_{j}}\left(t-a_{j}\right)\right]} \\
\times\left(k \sqrt{d_{j}} \sin \left[\sqrt{c_{j}}\left(t-a_{j}\right)\right] \sin ^{k-1}\left[\sqrt{d_{j}}\left(t-a_{j}\right)\right] \cos \left[\sqrt{d_{j}}\left(t-a_{j}\right)\right]\right. \\
\left.\left.\quad+(v+1) \sqrt{c_{j}} \sin ^{k}\left[\sqrt{d_{j}}\left(t-a_{j}\right)\right] \cos \left[\sqrt{c_{j}}\left(t-a_{j}\right)\right]\right)^{2}\right] d t>0
\end{aligned}
$$

where $\bar{q}_{2}(t)=\max \left\{-q_{2}(t), 0\right\}$, then all solutions of (3.3) are oscillatory.

If we choose $\Phi(t, s, l)=\rho(s)(t-s)^{\alpha}(s-l)^{\beta}$ for $\alpha, \beta>1 / 2$ and $\rho(s) \in C^{1}\left(\left[t_{0},(0, \infty)\right)\right)$, then we have that

$$
\varphi(t, s, l)=\frac{\rho^{\prime}(s)}{\rho(s)}+\frac{\beta t-(\alpha+\beta) s+\alpha l}{(t-s)(s-l)} .
$$

Thus by Theorems 2.8 and 2.9, we have two new oscillation results. 
Corollary 3.3. Suppose that, for any $T \geq 0$, there exist constants $a_{1}, b_{1}, a_{2}, b_{2}$ such that $T \leq a_{1}<$ $b_{1} \leq a_{2}<b_{2}$ and satisfies (2.1). For each $t \geq t_{0}$ and for some $\theta \geq 1$,

$$
\underset{t \rightarrow \infty}{\limsup } \int_{t_{0}}^{t} \rho^{2}(t)(t-s)^{2 \alpha}\left(s-t_{0}\right)^{2 \beta}\left[\rho(s) R(s)-\frac{\theta \rho(s) r(s)}{4}\left(2 \frac{\beta t-(\alpha+\beta) s+\alpha t_{0}}{(t-s)\left(s-t_{0}\right)}+\frac{\rho^{\prime}(s)}{\rho(s)}\right)^{2}\right] d s>0,
$$

for $j=1,2$, then (1.1) is oscillatory, where

$$
R(s)=M p(s)[1-a(\tau(s))] \frac{\tau(s)-\tau\left(a_{j}\right)}{s-\tau\left(a_{j}\right)}+a_{0}(1-a(s))|e(s)|^{k_{0}} \prod_{i=1}^{n} q_{i}^{k_{i}}(s),
$$

$a_{0}=\prod_{i=0}^{n} k_{i}^{-k_{i}}$, and $k_{0}, k_{1}, \ldots, k_{n}$ are positive constants satisfying $(a)$ and $(b)$ of Lemma 2.1.

Corollary 3.4. Suppose that, for any $T \geq 0$, there exist constants $a_{1}, b_{1}, a_{2}, b_{2}$ such that $T \leq a_{1}<$ $b_{1} \leq a_{2}<b_{2}$ and satisfies (2.23). Assume that there exists a function $\Phi \in Y$ such that, for each $t \geq t_{0}$ and for some $\theta \geq 1$,

$\limsup _{t \rightarrow \infty} \int_{t_{0}}^{t} \rho^{2}(t)(t-s)^{2 \alpha}\left(s-t_{0}\right)^{2 \beta}\left[\rho(s) \bar{R}(s)-\frac{\theta \rho(s) r(s)}{4}\left(2 \frac{\beta t-(\alpha+\beta) s+\alpha t_{0}}{(t-s)\left(s-t_{0}\right)}+\frac{\rho^{\prime}(s)}{\rho(s)}\right)^{2}\right] d s>0$,

for $j=1,2$, then (1.1) is oscillatory, where $\bar{R}(t)$ is defined by (2.25).

We say that a function $H=H(t, s)$ belongs to the function class $x$, if $H \in C\left(D, \mathbb{R}^{+}\right)$, where $D=\left\{(t, s): t_{0} \leq s \leq t<\infty\right\}$, which satisfies $H(t, t)=0, H(t, s)>0$ for $t>s$ and has partial derivatives $\partial H / \partial s$ and $\partial H / \partial t$ on $D$ such that

$$
\frac{\partial H}{\partial t}=h_{1}(t, s) \sqrt{H(t, s)}, \quad \frac{\partial H}{\partial s}=-h_{2}(t, s) \sqrt{H(t, s)},
$$

where $h_{1}(t, s), h_{2}(t, s)$ are locally integrable with respect to $t$ and $s$, respectively, in $D$.

If we choose $\Phi(t, s, l)=\sqrt{H_{1}(s, l) H_{2}(t, s)}$, for $H_{1}, H_{2} \in \mathcal{X}$, then we have that

$$
\varphi(t, s, l)=\frac{1}{2}\left(\frac{h_{1}^{(1)}(s, l)}{\sqrt{H_{1}(s, l)}}-\frac{h_{2}^{(2)}(t, s)}{\sqrt{H_{2}(t, s)}}\right)
$$

where $h_{1}^{(1)}(s, l), h_{2}^{(2)}(t, s)$ are defined as the following:

$$
\frac{\partial H_{1}(s, l)}{\partial s}=h_{1}^{(1)}(s, l) \sqrt{H_{1}(s, l)}, \quad \frac{\partial H_{2}(t, s)}{\partial s}=-h_{2}^{(2)}(t, s) \sqrt{H_{2}(t, s)} .
$$

Thus by Theorems 2.8 and 2.9, we also have the two following oscillation results. 
Corollary 3.5. Suppose that, for any $T \geq 0$, there exist constants $a_{1}, b_{1}, a_{2}, b_{2}$ such that $T \leq a_{1}<$ $b_{1} \leq a_{2}<b_{2}$ and satisfies (2.1). For each $t \geq t_{0}$ and for some $\theta \geq 1$,

$\underset{t \rightarrow \infty}{\limsup } \int_{t_{0}}^{t} H_{1}\left(s, t_{0}\right) H_{2}(t, s)\left[\rho(s) R(s)-\frac{\theta \rho(s) r(s)}{4}\left(\frac{h_{1}^{(1)}\left(s, t_{0}\right)}{\sqrt{H_{1}\left(s, t_{0}\right)}}-\frac{h_{2}^{(2)}(t, s)}{\sqrt{H_{2}(t, s)}}-\frac{\rho^{\prime}(s)}{\rho(s)}\right)^{2}\right] d s>0$,

for $j=1,2$, then (1.1) is oscillatory, where $h_{1}^{(1)}\left(s, t_{0}\right), h_{2}^{(2)}(t, s)$ are defined by (3.12),

$$
R(s)=M p(s)[1-a(\tau(s))] \frac{\tau(s)-\tau\left(a_{j}\right)}{s-\tau\left(a_{j}\right)}+a_{0}(1-a(s))|e(s)|^{k_{0}} \prod_{i=1}^{n} q_{i}^{k_{i}}(s),
$$

$a_{0}=\prod_{i=0}^{n} k_{i}^{-k_{i}}$, and $k_{0}, k_{1}, \ldots, k_{n}$ are positive constants satisfying $(a)$ and $(b)$ of Lemma 2.1.

Corollary 3.6. Suppose that, for any $T \geq 0$, there exist constants $a_{1}, b_{1}, a_{2}, b_{2}$ such that $T \leq a_{1}<$ $b_{1} \leq a_{2}<b_{2}$ and satisfies (2.23). Assume that there exists a function $\Phi \in \boldsymbol{y}$ such that, for each $t \geq t_{0}$ and for some $\theta \geq 1$,

$\limsup _{t \rightarrow \infty} \int_{t_{0}}^{t} H_{1}\left(s, t_{0}\right) H_{2}(t, s)\left[\rho(s) \bar{R}(s)-\frac{\theta \rho(s) r(s)}{4}\left(\frac{h_{1}^{(1)}\left(s, t_{0}\right)}{\sqrt{H_{1}\left(s, t_{0}\right)}}-\frac{h_{2}^{(2)}(t, s)}{\sqrt{H_{2}(t, s)}}-\frac{\rho^{\prime}(s)}{\rho(s)}\right)^{2}\right] d s>0$,

for $j=1,2$, then (1.1) is oscillatory, where $h_{1}^{(1)}\left(s, t_{0}\right), h_{2}^{(2)}(t, s)$ is defined by (3.12) and $\bar{R}(t)$ are defined by (2.25).

\section{Acknowledgments}

The aouthors thank the referees for their helpful comments to improve this paper. This research was supported by the NNSF of China (Grant no. 10771118) and NSF of Shandong (Grants no. ZR2009AM011 and no. ZR2009AQ010).

\section{References}

[1] M. S. Keener, "On the solutions of certain linear nonhomogeneous second-order differential equations," Applicable Analysis, vol. 1, no. 1, pp. 57-63, 1971.

[2] S. M. Rankin III, "Oscillation theorems for second-order nonhomogeneous linear differential equations," Journal of Mathematical Analysis and Applications, vol. 53, no. 3, pp. 550-553, 1976.

[3] H. Teufel Jr., "Forced second order nonlinear oscillation," Journal of Mathematical Analysis and Applications, vol. 40, pp. 148-152, 1972.

[4] Y. G. Sun, "A note on Nasr's and Wong's papers," Journal of Mathematical Analysis and Applications, vol. 286, no. 1, pp. 363-367, 2003.

[5] A. H. Nasr, "Necessary and sufficient conditions for the oscillation of forced nonlinear second order differential equations with delayed argument," Journal of Mathematical Analysis and Applications, vol. 212, no. 1, pp. 51-59, 1997. 
[6] J. S. W. Wong, "Second order nonlinear forced oscillations," SIAM Journal on Mathematical Analysis, vol. 19 , no. 3, pp. 667-675, 1988.

[7] D. Çakmak and A. Tiryaki, "Oscillation criteria for certain forced second-order nonlinear differential equations with delayed argument," Computers $\mathcal{E}$ Mathematics with Applications, vol. 49, no. 11-12, pp. 1647-1653, 2005.

[8] A. H. Nasr, "Sufficient conditions for the oscillation of forced super-linear second order differential equations with oscillatory potential," Proceedings of the American Mathematical Society, vol. 126, no. 1, pp. 123-125, 1998.

[9] A. G. Kartsatos, "On the maintenance of oscillations of $n$th order equations under the effect of a small forcing term," Journal of Differential Equations, vol. 10, pp. 355-363, 1971.

[10] Q. Kong, "Interval criteria for oscillation of second-order linear ordinary differential equations," Journal of Mathematical Analysis and Applications, vol. 229, no. 1, pp. 258-270, 1999.

[11] J. S. W. Wong, "Oscillation criteria for a forced second-order linear differential equation," Journal of Mathematical Analysis and Applications, vol. 231, no. 1, pp. 235-240, 1999.

[12] D. Çakmak and A. Tiryaki, "Oscillation criteria for certain forced second-order nonlinear differential equations," Applied Mathematics Letters, vol. 17, no. 3, pp. 275-279, 2004.

[13] Y. G. Sun and J. S. W. Wong, "Oscillation criteria for second order forced ordinary differential equations with mixed nonlinearities," Journal of Mathematical Analysis and Applications, vol. 334, no. 1, pp. 549-560, 2007.

[14] Y. G. Sun and F. Meng, "Interval criteria for oscillation of second-order differential equations with mixed nonlinearities," Applied Mathematics and Computation, vol. 198, no. 1, pp. 375-381, 2008.

[15] C. H. Ou and J. S. W. Wong, "Forced oscillation of $n$ th-order functional differential equations," Journal of Mathematical Analysis and Applications, vol. 262, no. 2, pp. 722-732, 2001.

[16] Y. G. Sun and R. P. Agarwal, "Forced oscillation of $n$ th-order nonlinear differential equations," Functional Differential Equations, vol. 11, no. 3-4, pp. 587-596, 2004.

[17] Y. G. Sun and R. P. Agarwal, “Interval oscillation criteria for higher-order forced nonlinear differential equations," Nonlinear Functional Analysis and Applications, vol. 9, no. 3, pp. 441-449, 2004.

[18] Q. Yang, "Interval oscillation criteria for a forced second order nonlinear ordinary differential equations with oscillatory potential," Applied Mathematics and Computation, vol. 135, no. 1, pp. 49-64, 2003.

[19] X. Yang, "Forced oscillation of $n$ th-order nonlinear differential equations," Applied Mathematics and Computation, vol. 134, no. 2-3, pp. 301-305, 2003.

[20] J. S. W. Wong, "On Kamenev-type oscillation theorems for second-order differential equations with damping," Journal of Mathematical Analysis and Applications, vol. 258, no. 1, pp. 244-257, 2001.

[21] A. Tiryaki, D. Çakmak, and B. Ayanlar, "On the oscillation of certain second-order nonlinear differential equations," Journal of Mathematical Analysis and Applications, vol. 281, no. 2, pp. 565-574, 2003.

[22] Y. V. Rogovchenko and F. Tuncay, "Oscillation criteria for second-order nonlinear differential equations with damping," Nonlinear Analysis: Theory, Methods \& Applications, vol. 69, no. 1, pp. 208221, 2008.

[23] R. P. Agarwal, S. R. Grace, and D. O'Regan, Oscillation Theory for Second Order Linear, HalfLinear, Superlinear and Sublinear Dynamic Equations, Kluwer Academic Publishers, Dordrecht, The Netherlands, 2002.

[24] R. Xu and F. Meng, "New Kamenev-type oscillation criteria for second order neutral nonlinear differential equations," Applied Mathematics and Computation, vol. 188, no. 2, pp. 1364-1370, 2007.

[25] E. F. Beckenbach and R. Bellman, Inequalities, Ergebnisse der Mathematik und ihrer Grenzgebiete, Springer, Berlin, Germany, 1961. 


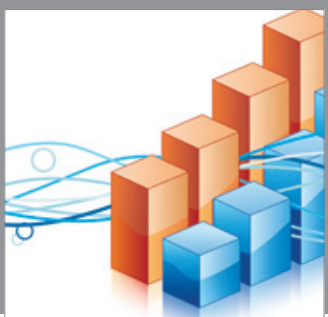

Advances in

Operations Research

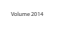

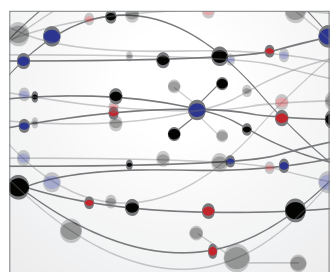

\section{The Scientific} World Journal
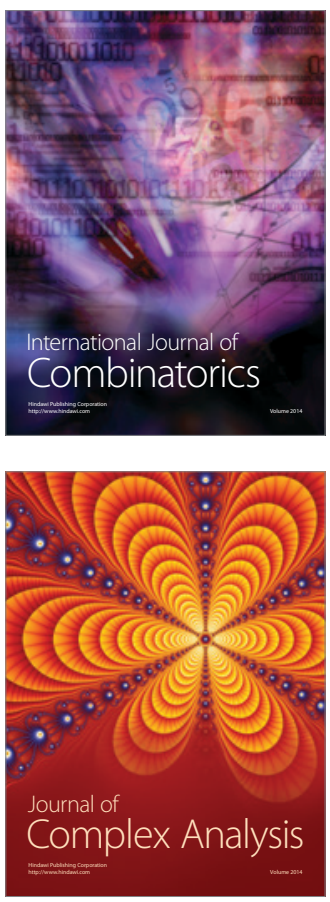

International Journal of

Mathematics and

Mathematical

Sciences
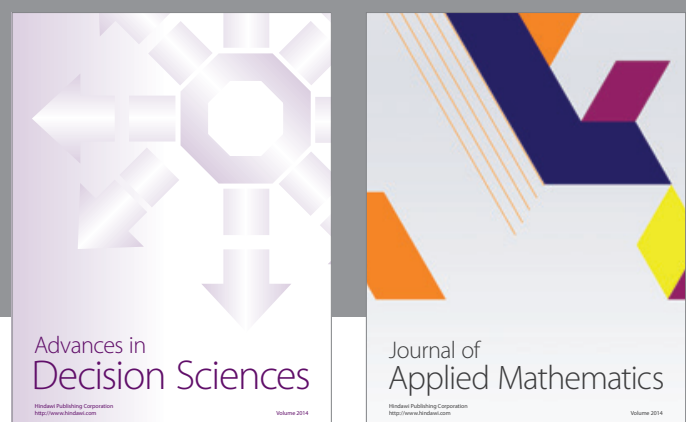

Journal of

Applied Mathematics
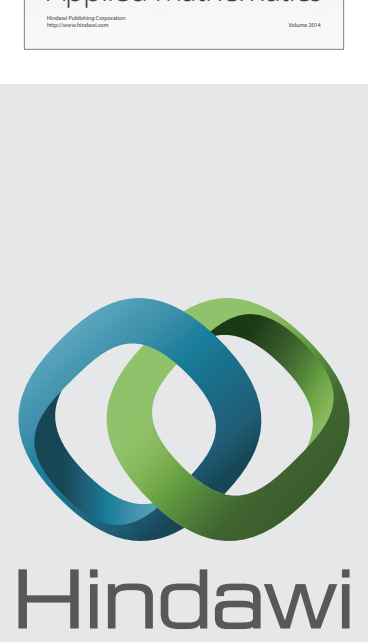

Submit your manuscripts at http://www.hindawi.com
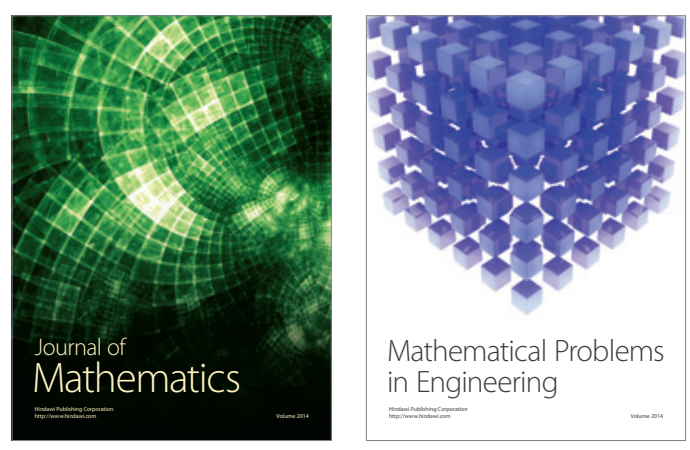

Mathematical Problems in Engineering
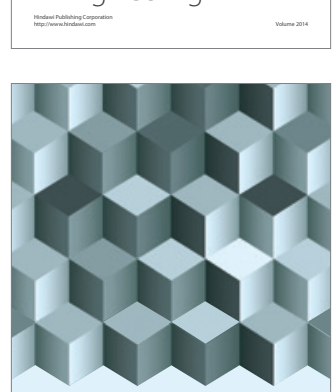

Journal of

Function Spaces
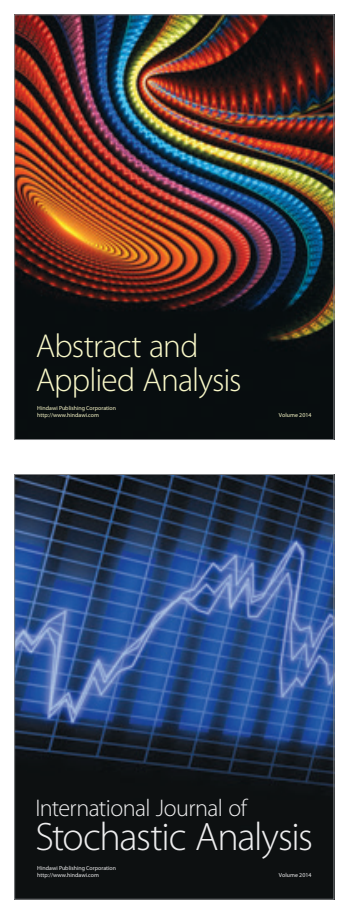

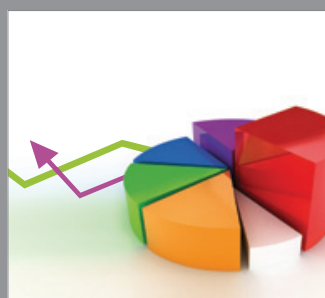

ournal of

Probability and Statistics

Promensencen
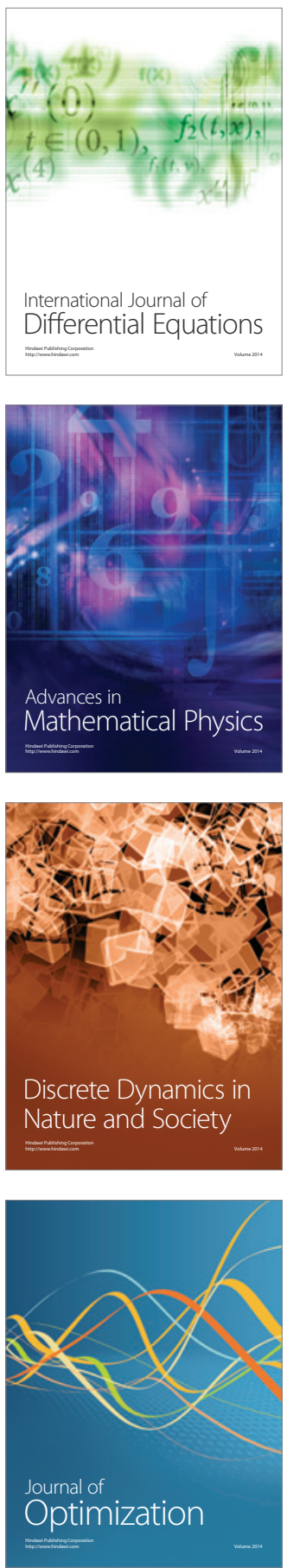